\title{
STABILITY ROUTING IN FSO-MANETS
}

\author{
Deena George ${ }^{1}, S_{\text {Perumal Sankar }}^{2}$ \\ ${ }^{I} M$ Tech, ECE, TIST, Kochi, Kerala, India \\ ${ }^{2}$ Professor, ECE, TIST, Kochi, Kerala, India
}

\begin{abstract}
Routing in MANET is one of the most researched areas in the field of networking; moreover it is one of the most complex tasks. $R F$ based transmission techniques are widely used in wireless communication networks. Due to the availability of sophisticated optical components, the networking domain could be merged with optical domain to have a higher capacity and better transmission. So in order to overcome the disadvantages of RF domain, we can use light as a medium between nodes. i.e, FSOMANET. This work is aimed at designing an efficient routing in FSO MANET. This paper proposes a method to find the stable path as well as stable nodes between the source and destination. The steps are as follows. i) Topology Management ii) Trust Level Calculation iii) Award and Reward ranking iv)Path Selection. The proposed technique will be implemented in the working platform of MATLAB.
\end{abstract}

Keywords: Mobile Ad Hoc Network, Free Space Optics, Link Stability, Delay Tolerant Networking, Clustering Technique, Award and Reward

\section{INTRODUCTION}

Free space optics is a line of sight technology which uses air as a medium between transmitter and receiver. In 1960 the FSO technology was developed for military applications[3]. At the beginning of the $21^{\text {st }}$ centuary FSO communication started to see considerable shares in commercial telecommunication systems. The major advantage of FSO is high bandwidth and no spectrum licensing. Free-space optics (FSO) is a technology for transmitting data using optical signals propagating through the air.

Mobile Adhoc Networks (MANETs) is an emerging technology that allows establishing instant communication infrastructures for military and civilian applications [11]. MANET is a network architecture that can be rapidly deployed without pre-existing fixed network infrastructure. Usually nodes in a MANET can frequently join and leave the network, often without warning, and without any disruption to other node's communication.

Mobile ad hoc network (MANET) also called short lived network can be defined as a type of ad hoc network that can change locations and configure itself randomly. Because MANETS are mobile, they use wireless connections to connect to various networks. This can be a Wi-Fi connection, or another medium, such as a cellular or satellite communication. It can also be defined as a collection of mobile nodes communicating in a multi-hop way without any fixed infrastructure such as access points or base stations. Simply stating, a Mobile Ad hoc Networks (MANET) is one that comes together when required, but it is not necessary that it requires support from existing Internet infrastructure or any other kind of fixed stations. Formally, an ad hoc network can be defined as an autonomous system of mobile hosts (also serving as routers) connected by wireless links, which leads to the formation of a communication network.

Due to the continuous change in network topology, analyzing the stable path between source and destination is one of the important issues in adhoc networks. And also interference from other light sources effects the transmission. So in order to overcome this we propose a new method for finding the path between source and destination. The paper is organized as follows; Section 2 introduces the concept of stability. Section 3 discusses the stability analysis methodology. Finally the concluding remarks are given in section 4.

\section{STABILITY}

In FSO-MANET, the mobile nodes are continuously moving. So the shortest path is not the safest path in the network. If we are not considered the stability of the path, the link may be broken. Link stability indicates not only how stable the link is and also shows how long it can support communications between two nodes. In this paper we propose a new method for finding the [7] stable path as well as stable nodes with the help of award and reward scheme. This will provide a better path between two end points. So the connectivity problems can be avoided.

\section{METHODOLOGY}

An adhoc network consists of some thousands of sensor nodes that are deployed over a geographic area for monitoring the environment which have the ability to communicate among them. In a geographic area the nodes are deployed randomly. For an effective transmission we need a clustering approach. The communication between base station and sensor nodes can take place either in flat fashion or hierarchial fashion. In hierarchial architecture 
which is also known as clustering, sensor nodes are first grouped into a cluster. In cluster based architectures, mobile nodes are divided into virtual groups. Each cluster has various adjacent clusters. All the clusters have the same rules. A cluster can be made up of a Cluster Head node and Cluster Members. Here, Cluster Head nodes are used for controlling the cluster and the area of the cluster is usually about one or two hops from the Cluster Head node. There are many cluster based architectures. Clustering schemes can be classified according to various criteria. For example, they can be classified according to architectures dependency on Cluster Head or on Non Cluster Head. The first architecture requires a Cluster Head for controlling and managing the group, and the second one does not have a particular node to perform this task. The other way to classify the cluster-based architectures is observing the hop distance between node pairs in a cluster [10].

For this cluster-head selection procedure we are using DTN (Delay Tolerant Networking) approach. Delay-tolerant networking (DTN) is an approach to computer network infrastructure that seeks to address the technical issues in heterogeneous networks that may cause lack of continuous network connectivity. Examples of such networks are those operating in mobile or terrestrial environments or planned networks in space. DTNs have the potential to interconnect devices in regions where current networking technology cannot reach. The idea is that an end-to-end connection may never be present. To make transmission of packets possible, intermediate nodes take custody of the data being transferred and forward it as the opportunity arises. Both links and nodes may be inherently unreliable and disconnections may be long-lived. To realize the DTN vision, path must be found over multiple unreliable, intermittently connected hops. In this paper a novel routing approach is developed for the DTNs, the proposed approach comprises of four phases they are topology management, trust level calculation, award-reward setting and path selection. The architecture of the proposed approach is given in figure below,

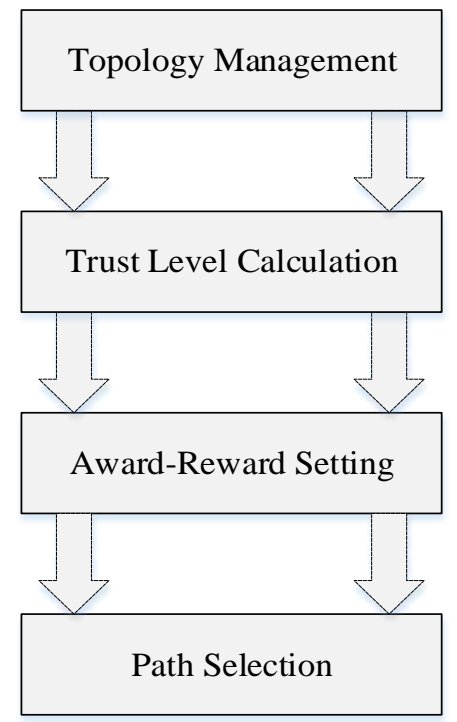

Figure 1: Process follows the proposed method
In DTNs proving constant link between nodes is very complex task, hence in this proposed system a virtual topology is formed and based on this topology further routing procedure is carry out. The k-means algorithm is applied to maintain the topology and the topology is formed based on the distance. Then the trust level of every node is determined by sending and receiving sample packets. Subsequently based on the trust level the award-reward value is defined for the nodes then based on this value the routing path is selected.

\subsection{Topology Management}

In DTN to realize the management of the overall delivery topology and the formation of specific delivery graphs the topology management is employed. In the proposed method $\mathrm{k}$-means algorithm is applied for the topology management. $\mathrm{K}$-means algorithm is a soft computing Clustering technique which groups the nodes or objects based on the similarity. Clustering is a method of grouping a set of objects in such a way that objects in the same group are more similar to each other than to those in other groups (Clusters). In general the Cluster in formed based any one or more attributes of the particular objects. In our proposed method the Cluster is not made of any object instead of that the nodes are grouped in DTN. The attribute we are considering here to group the node is distance and energy. Hence in this paper the nodes are Clustered based on the distance between the nodes, i.e. the shortest distance nodes with closer energy are kept in a same Cluster. In every Clustering operation the selection of Cluster is one of the challenging tasks. The effectiveness of Cluster is completely depends on the Cluster head. In the proposed method we are using the k-means algorithm for the selection of Cluster head as well as for the Clustering.

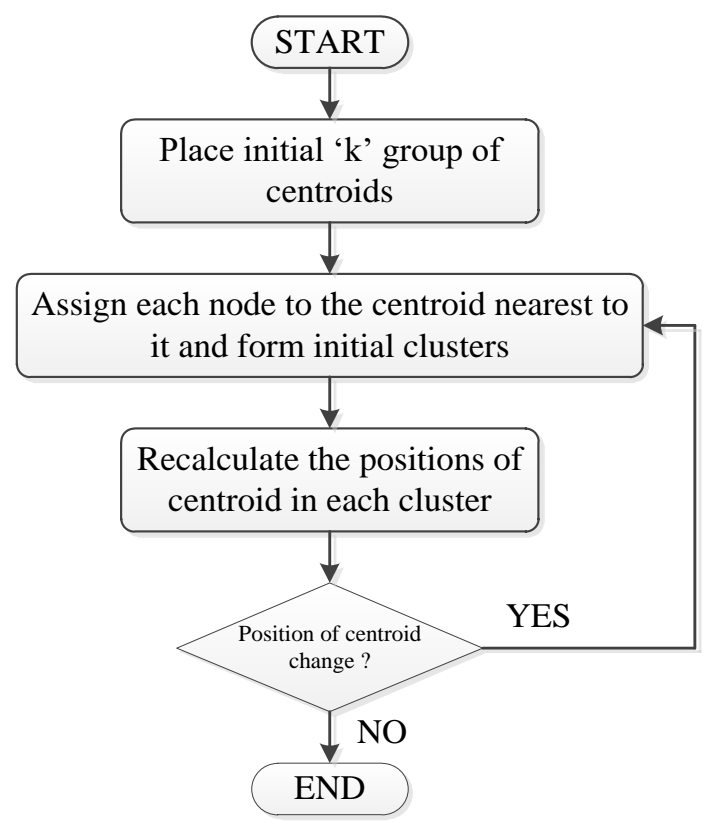

Figure 2: Flow chart for clustering the nodes in DTN

The Clustering algorithm can group the nodes in the nearby region in a single group and the Cluster head is assumed as a base node, so that the connection can provide easily. Then 
the trust level for every node in DTN has to calculate it is discussed in the subsequent section.

\subsection{Trust Level Calculation}

In this phase the trust ability of every node in the DTN, it ensure the denial-of-service (DoS) attach. The DoS attack is an attempt to make device or network resource unavailable to its intended users. A DoS attack generally consists of efforts to temporarily or indefinitely interrupt or suspend services of a host connected to the Internet. Hence in order to ensure the trust ability of a node in DTN, a sample set of packets is sent and receive it back by the same node, the sending and receiving ratio represents the trust level of the particular node. For example, consider two node A and B, here A wants to verify the trust level of the node B means, initially the node sent a set of sample packets to $B$, then the node $B$ retransmit the sample packets to $B$. If the number of packets transmitted by $A$ and received from the $B$ is same then the trust level of node B is $100 \%$. In our work the center node is consider as the base node, so that it alone send sample packets to its group node for calculating the trust level [12].

\subsection{Award-reward Setting}

In general term the award and reward is a kind of appreciation, to motivate and our best participant in everywhere. In this paper the award and reward is a kind of ranking based on the trust ability of nodes in DTN.

For example if the nodes N1, N2, N3, N4, N5, N6, N7, N8, N9 and N10 having AR values as 10, 9, 8, 7, 6, 5, 4, 3, 2 and 1 respectively, then the award-reward setting become A5, A4, A3, A2, A1, R1, R2, R3, R4 and R5 respectively, where A5 means Award-5 and R5 means Reward-5. Hence the highest awarded node is highly trusted for routing and highest rewarded node is never considered for routing.

\subsection{Path Selection}

Selection of path is one of the crucial processes in every network and it became a challenging task in DTN. In the proposed system the path is selected on the basis of awardreward setting. The operation of path selection based on the proposed approach is shown in figure.

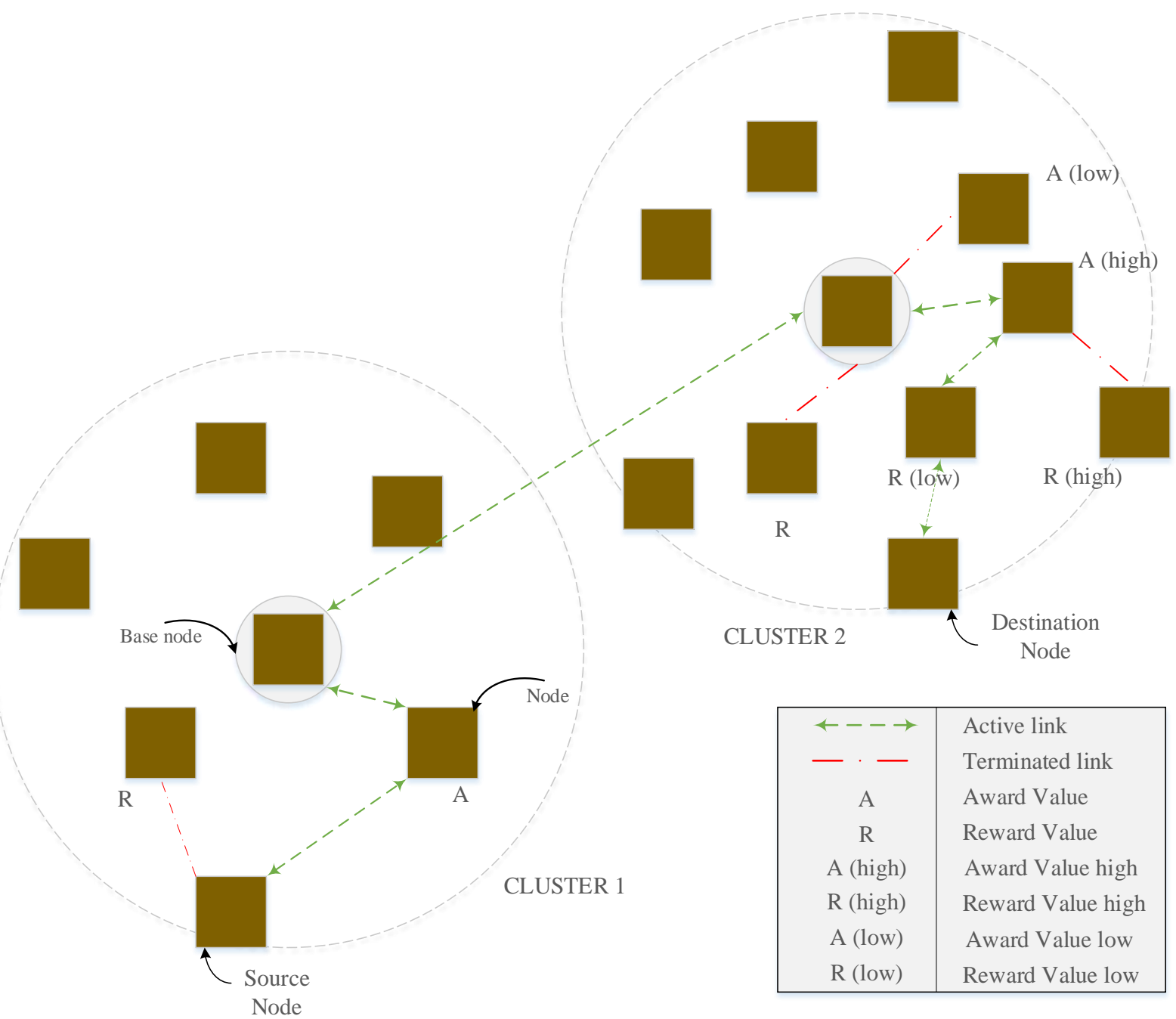

Figure 3: Path Selection 
The path selection is a major process in the DTN so in the proposed system the path is selected based on the awardreward setting. Initially the nodes in the DTN are grouped based on the distance and the trust value for every node is calculated by sending a sample packets. Then based on the trust level the award-reward value is provided for every nodes and this value is consider for the path selection the operation of the path selection is shown in figure 3. Let us consider a DTN having 18 nodes; initially it is clustered into two by applying k-means algorithm, so the Cluster1 contain 7 nodes and Cluster2 contains 11 nodes. A node from Cluster1 is considered as a source node and node in Cluster2 is considered as destination shown in figure 3. First the source node sends the information to its base node and it forward it to the corresponding base node, then the corresponding base node convey the information to destination node through the award-reward procedure. For sending the information from source to its base it analyzes the award-reward value of its adjacent node and the appropriate node is selected. The source nodes never send directly to its base unless it is in adjacent. Here the source node check the award-reward value of adjacent, where two nodes are adjacent to the source among them one have reward value and another has award value, so the awarded node is selected. Base node is adjacent to the selected node, so it directly sends the information to the base.

After receiving the information by the base node it analyses the destination address, that whether the destination is also present in its group otherwise it transmit the information to the adjacent base nodes, then the process is repeated to reach the destination in Cluster2 shown in figure3. The format of the packet is shown in figure 4.

\begin{tabular}{|l|l|l|}
\hline Header & Information & Trailer \\
\hline
\end{tabular}

Figure 4: Packet format

The Header and Trailer are fixed size and having the sender and destination address. The nodes in the DTN receive the packet and check whether the destination address is same as its address otherwise it transmits it to the base node. The base node checks whether the destination node exists in its group otherwise transmit it to the other group. While selecting the path based on the award-reward values the loss can minimized by preventing from the DoS attacks. Moreover the path is in short rage so the continuity can maintain for a long time than the normal DTN. Hence form this overall system the proper routing can achieved in DTN, by enhancing the security and reducing the loss.

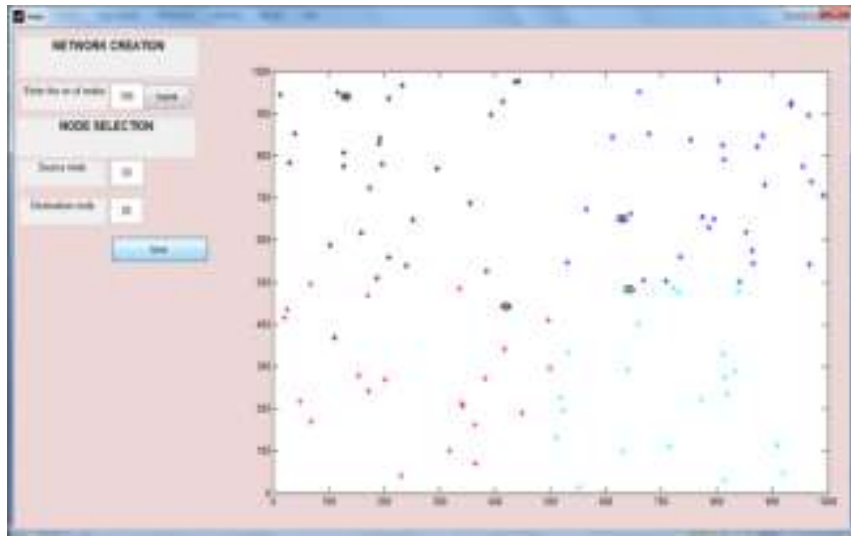

Figure 5: Simulation Results in MATLAB

The figure 5 shows the network scenario which is created in MATLAB and analyze the path which is more stable based on the trust value and award reward calculation.

\section{CONCLUSION}

Due to the frequent changes in network topology, in FSOMANET networks to identify the stable path is one of the important issues in the network. In this paper we introduce a new method to identify the stable path as well as stable nodes with the help of Delay Tolerant Networking. This method helps to find out the suitable path based on trust value and award reward calculation process. We have simulated the network scenario in MATLAB and identify the suitable path. From this paper we can concluded that the award and reward scheme provides the better solution to avoid the connectivity issues in FSO-MANET networks. So we can transmit the information without any connectivity problems.

\section{REFERENCE}

[1] Matthias R. Brust, Steffen Rothkugel, and Adrian Andronache, "Node Stability in Dynamic Communication Networks". in Proceedings of the First Asia International Conference on Modelling \& Simulation (AMS'07)0-76952845-7/07 \$20.00 @ 2007

[2] Sudip Misra, Sanjay K. Dhurandher, Mohammad S. Obaidat, "Node Stability-Based Location Updating in Mobile Ad-Hoc Networks".in ieee systems journal, Vol. 2, No. 2,June 2008

[3]. Priyanka Jangir and Bhuperdra Suman "Survey on Performance of Free Space Optical Communication Links under Various Field Parameters" e-ISSN: 2278-1676,pISSN: 2320-3331, Volume 9, Issue 2 Ver. V (Mar - Apr. 2014), PP 71-75

[4] Navid Nikaein, Christian Bonnet, "Improving Routing and Network Performance in Mobile Ad Hoc Networks Using Quality of Nodes". https://hal.inria.fr/inria-00466593 Submitted on 24 Mar 2010.

[5] Mandeep Kaur Gulati and Krishan Kumar, "Survey of Stability Based Routing Protocols in Mobile Ad-hoc Networks" International Journal of Computer Science Engineering and Technology( IJCSET)2011

[6] T. Rajamohan Reddy and N. Sobharani“" Selective ondemand protocol for finding reliable nodes to form stable 
paths in ADHOC networks". International Journal of Advanced Research in Computer Engineering \& Technology Vol 1, Issue 5, July 2012.

[7]P. I. Basarkod, S. S. Manvi, D.S.Albur , "Mobility Based Estimation of Node Stability in MANETs." 2013 IEEE International Conference on Emerging Trends in Computing, Communication and Nanotechnology (ICECCN 2013).

[8] Devideen ahirwar and Prof. Sarvesh Singh Rai, "Improvement of AODV Routing Protocol Algorithm with Link Stability and Energy Efficient Routing for MANET". International Journal of Computer Science Engineering and Technology( IJCSET)

[9] G.Madhukar Rao and ,T.santhosh, " Quality of service Routing Using Stable Nodes in Mobile Ad hoc Networks". ISSN (e): 2250 - 3005, Vol, 04 , Issue, 11 . November 2014.

February 2014 .

[10].Lloret, Jaime,Miguel Garcia,Diana Bri, and Juan R Diaz." A Cluster Bases Architecture to Structure the Topology of Parallel Wireless Sensor Networks".

[11]Yanhui Chen. "Performane evaluation of modified IEEE 802.11 MAC for multi-hop adhoc network", $17^{\text {th }}$ internaqtional conference on Advanced Information Networking and Applications 2003 AINA 2003 AINA03,2003

[12] Advances in Intelligent Systems and Computing,2012 